Aim The intervention aims to reduce the resultant distress from breathlessness whilst improving functional capacity with improving the activities of daily living ability and the overall quality of life.

Method The intervention was compiled using available evidence from published data specifically geared at refractory breathlessness in advanced disease.

Results The group developed a set of objectives, referral criteria and a service specification to replicate across the organisation. Evidence from the literature supports carers managing the "dyspnoea crisis" and inclusion with exploration of their feelings, concerns and expectations and education. Recent work suggested that low intensity (one session) breathlessness management was as effective as high (three sessions) intensity but the wider remit of this intervention and its proposed outcomes, three sessions were employed.

Sessions comprise of components of education with regard to physiological changes that occur during breathlessness, why exercise is important and why breathing control works. Anxiety management including the carer is a fundamental component in managing the dyadic units response to "dyspnoea crisis". Finally goal setting in terms of the 3Ps (pacing, planning and prioritising) are key to enabling functional improvement. Objective and subjective assessments are completed to help track changes over time for patients/carers and professionals.

Conclusions This breathlessness management intervention has been clearly articulated in a pathway to help internal and external professionals understand the process and the components involved whilst meeting the need for commissioning bodies to see evidence of activity linked to meaningful patient outcomes.

\section{P-116 ANXIETY MANAGEMENT IN PRACTICE; DEVELOPING A RELAXATION CD}

Sharon Penny, Gill Thomas, Belinda Hitchens. Princess Alice Hospice, Esher, UK

\subsection{6/bmjspcare-2016-001245.139}

Background The impact of living with a life-limiting illness and the effects of living with physical and psychological symptoms and impact can create high levels of anxiety for both the patient and their carer. The most common issues that can exacerbate anxiety are breathlessness, pain, fatigue and the anticipation of what the future holds.

An occupational therapist, complementary therapist and hypnotherapist provide supportive interventions to patients and carers. Evaluation of these services reports a positive impact of anxiety reduction after sessions but this often reduces over time.

The therapists were eager to find ways of increasing the efficacy of these sessions and support the individuals to feel empowered to support themselves outside their sessions using the techniques gained.

Aims To produce a relaxation CD with different styles of relaxation to support and develop their ability to relax in their everyday lives.

Method A search established what relaxation CDs were available There was no appropriate relaxation CD with mixed relaxation styles available. We focused on four approaches:

- Guided imagery

- Progressive muscular relaxation

- Autogenic relaxation

- Mindfulness.
A local composer offered to record our own CD for free. From experience we knew some people prefer music and others only the spoken word. We piloted the CD with 20 people. Overall the feedback was positive with some modifications suggested. Findings Two people didn't like the CD but the others continued to use the $\mathrm{CD}$ regularly reporting a reduction in insomnia, breathlessness and anxiety.

Conclusions The CD has been very well received and has been reported to improve relaxation using a non-invasive approach.

Implications for practice The CD is now used in the breathlessness group, with our patients and carers and is available on the ward for patients and staff and for sale in our shops.

\section{P-117 "BREATHE BETTER": EXPLORING THE POTENTIAL IMPACT OF SUPPORT GROUPS FOR PEOPLE WITH END- STAGE COPD}

1,2 Laura Green, 'Clare Rayment, ${ }^{3}$ George Lawson. 'Marie Curie Hospice Bradford, Bradford, UK; ${ }^{2}$ University of Bradford, Bradford, UK; ${ }^{3}$ University of Leeds, Leeds, UK

\subsection{6/bmjspcare-2016-001245.140}

Background The Breathe Better group (BBG) has been running from Marie Curie Hospice Bradford (MCHB) since 2008. It aims to reduce dyspnoea, improve quality of life and mastery of illness for patients with end stage COPD. It uses four methods: education, exercise, pharmacological management and individual consultations. We aimed to ascertain the impact of the BBG.

Method A retrospective longitudinal study was performed on patients referred between August 2010 and June 2013. Data were collected on shuttle walk distance, mastery, Hospital Anxiety and Depression Scores (HADS), advance care planning (ACP), and mortality. Outcomes were analysed using a paired t-test.

Results Data from 56 patients was analysed. Mean shuttle walk distance increased by an average of $10.8 \mathrm{~m}$ which was statistically significant $(\mathrm{p}=0.05)$. Total HADS score improved by 2 points. $65 \%$ of patients had an ACP after the group, compared with $24 \%$ prior to commencement. $76 \%$ patients died with two years of the group. Place of death (below) demonstrates an increase in rates of hospice death when compared with regional and national COPD data.

Place of Death $(\mathrm{n}=45)$

\begin{tabular}{ll}
\hline Care home & $\mathbf{1 1 \%}$ \\
\hline Home & $7 \%$ \\
Hospital & $38 \%$ \\
Hospice & $31 \%$ \\
Not recorded & $13 \%$ \\
\hline
\end{tabular}

Discussion Results suggest that BBG improves shuttle walk distance and recording of ACP which appears to influence place of death. Missing data means conclusions are based on a small sample size. Improved data recording is a local priority in order to render future evaluations more meaningful.

Implications Other hospices may wish to consider pilot schemes especially as often this patient group are unable to access current NHS schemes such as Pulmonary Rehabilitation due to their poor health. A larger study with a control group of patients who did not attend will allow more accurate evaluation. 